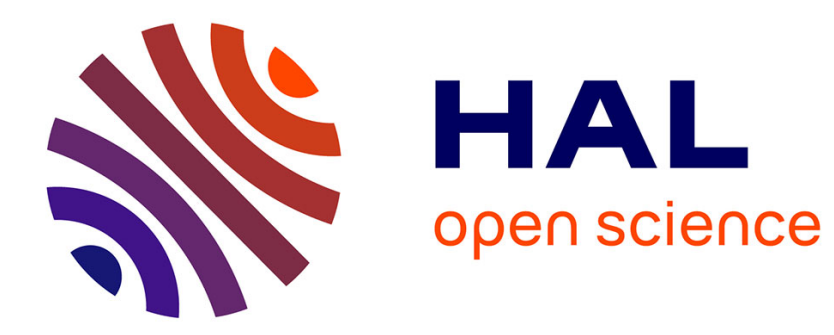

\title{
Sur la dynamique des acquisitions à l'école élémentaire
} Alain Mingat

\section{To cite this version:}

Alain Mingat. Sur la dynamique des acquisitions à l'école élémentaire. Revue Française de Pédagogie, 1987, 79. hal-03201606

\section{HAL Id: hal-03201606 https://hal.science/hal-03201606}

Submitted on 4 Jun 2021

HAL is a multi-disciplinary open access archive for the deposit and dissemination of scientific research documents, whether they are published or not. The documents may come from teaching and research institutions in France or abroad, or from public or private research centers.
L'archive ouverte pluridisciplinaire HAL, est destinée au dépôt et à la diffusion de documents scientifiques de niveau recherche, publiés ou non, émanant des établissements d'enseignement et de recherche français ou étrangers, des laboratoires publics ou privés.

\section{(a)(1) $\$$}

Distributed under a Creative Commons Attribution - NonCommercial - NoDerivatives| 4.0 


\section{SUR LA DYNAMIQUE DES ACQUISITIONS À L'ÉCOLE ÉLÉMENTAIRE}

\author{
par A. MINGAT
}

II a été montré qu'à l'issue de la classe de $C P$, les Jèves ont des niveaux d'acquisitions scolaires très variés. Ce résultat en lui méme est banal. Ce qui l'est moins, c'est qu'une part substantielle des différences interindividuelles d'acquisitions tient à l'école elle-même (en raison de différences dans la pédagogie mise en couvre et de la relativement forte variabilité de l'efficacité pédagogique des maîtres). Face à ce constat, il est d'un intérét évident, et c'est là l'objectif de cet article, de s'interroger sur les conséquences que cela a sur les acquisitions ultérieures de l'élève au cours de sa scolarité primaire. Deux hypothèses (au moins) peuvent être formulées: la première met l'accent sur le caractère cumulatif des acquisitions et sur le fait que de bien maîtriser certains concepts et de bien posséder certaines connaissances sont des éléments facilitants pour les nouveaux apprentissages. La seconde, au contraire, souligne que les caractéristiques personnelles de l'élève et l'évolution de sa maturité sont des facteurs qui déterminent la capacité à apprendre, le fait qu'on ait appris un peu plus ou un peu moins à un moment donné étant transitoire et finalement de peu d'importance. L'analyse statistique, menée à partir d'une enquête longitudinale effectuée dans l'agglomération dijonnaise, conclut très clairement que la première hypothèse est corroborée par les faits, les écarts en fin de CP qui dérivent d'une plus ou moins grande efficacité de l'école loutre les écarts qui tiennent aux caractéristiques personnelles des élèves) ayant des effets rémanents assez substantiels au cours de la scolarité ultérieure.

\section{I. - INTRODUCTION}

La question abordée dans cet article (1) se rattache au probleme général de la relation entre le processus de développement cognitif personnel de l'enfant et celui des acquisitions de connaissances scolaires en tant quie résultat des activités d'enseignement. Nous nous concentrerons sur un aspect particulier de ce problème général en nous interrogeant sur le point de savoir si on doit observer des effets permanents ou différès d'activités pédagogiques spécifiques menées à un moment donné du cursus éducatif.

Pour présenter cette question de façon schématique, imaginons qu'une activité pédagogique spécifique produise des "plus " ou des "moins" par rapport à ce qu'on aurait pu attendre comme résultat d'une activité pédagogique standard pour des élèves de caractéristiques personnelles données, ce résultat étant évalué en termes d'acquisitions scolaires. La question est alors de savoir si ces résultats obtenus en un point donné du cursus éducatif constituent des avantages (ou des handicaps) pour les acquisitions futures.

Deux hypothèses alternatives (au moins) existent en effet sur ce point. La première souligne que les acquisitions réalisées à un moment donné sont des " facteurs de production "importants pour les acquisitions ultérieures et que le fait de bien maîtriser certains concepts et de posséder certaines connaissances sont des éléments facilitant pour les nouveaux apprentissages. La seconde met l'accent sur le caractère fondamentalement artificiel et transitoire des écarts réalisés. Ce qui est important, ce sont les caractéristiques personnelles de l'élève et l'évolution de sa maturité. Ces éléments déterminent la capacité à apprendre et les écarts marginaux d'acquisitions à un moment donné sont à cet égard de peu d'importance. Dans cette même ligne d'arguments, certains suggèrent que les apprentissages "forcès" pourraient même exercer un impact négatif sur les possibilités ultérieures d'apprendre.

Pour explorer la pertinence de ces deux hypothèses alternatives, deux méthodes peuvent être envisagées. La première consiste à rechercher dans les constructions théoriques existantes les arguments en faveur de l'une ou de l'autre. Cela suppose que les constructions théoriques soient suffisamment corroborees par ailleurs par les faits ou qu'on ait suffisamment confiance en elles d'une part et 
qu'on juge leur domaine d'application pertinent pour le type de problème posé d'autre part. La seconde consiste à tester directement la capacité empirique des deux hypothèses dans des situations concrètes particulières. On a alors une conception externe ou poppérienne de la validité des hypothèses dans la mesure où plutôt que de discuter des théories ou des hypothèses elles-mêmes, on fait porter le test empirique sur leurs conséquences observables (2).

Ces deux voies peuvent bien sûr être mises en œuvre de façon complémentaire sachant que si la première a des vertus évidentes, la seconde ne saurait être ignorée car c'est in fine de la capacité des théories à rendre compte de la réalité que dépend leur intérêt et leur survie. C'est la seconde voie, celle de l'épreuve externe, qui a été suivie dans ce texte.

Pour la mettre en œuvre, il convient par conséquent de choisir des situations concrètes dans lesquelles les faits d'observation pourraient réfuter l'une ou l'autre des constructions théoriques mises à l'épreuve. On notera toutefois que cette méthode ne permet pas de valider, au sens fort du terme, une hypothèse mais seulement de la réfuter car il n'y a pas symétrie entre validation et réfutation. Pour reprendre l'exemple de K. Popper, il suffit de voir un seul corbeau blanc pour réfuter la proposition selon laquelle tous les corbeaux sont noirs, alors que l'observation d'un corbeau noir (ou même d'un nombre très grand de corbeaux de la même couleur) ne fait qu'augmenter le degré de corroboration empirique de la proposition sans jamais véritablement la vérifier.

Les situations concrètes qui ont été choisies concernent les acquisitions scolaires à l'école èlémentaire. Les activités pédagogiques spécifiques de référence concernent le cours préparatoire et on examine leur impact sur les acquisitions scolaires des élèves telles qu'on peut les apprécier deux années après, c'est-à-dire en fin de Cours élémentaire deuxième année. Les données analysées sont celles d'une enquête longitudinale engagée dans 16 écoles primaires de la banlieue dijonnaise à la rentrée scolaire de septembre 1982 dans le but d'évaluer les résultats d'une action Zone d'Education Prioritaire au Cours préparatoire (3). Deux aspects de l'activité pédagogique sont ici considérés :

1. L'action ZEP au CP reposait notamment sur l'idée que certains enfants possédaient insuffisamment les prérequis nécessaires pour les acquisitions fondamentales et qu'il convenait par conséquent de développer des activités spécifiques visant à compenser ce handicap initial. A raison de l'équivalent d'une journée par semaine, les activités courantes du CP ètaient mises entre parenthèses pour faire place à des activités de psycho-motricité ou de développement du langage selon ce qui apparaissait le plus approprié au cas de chaque enfant. Ces activités concernaient environ la moitié des enfants des classes ZEP, alors qu'il était proposé à l'autre moitié (ceux qui étaient supposés ne pas avoir de besoins particuliers de ces types) des activités sans contenu directement scolaire (travaux manuels, activités de groupe...). A la fin de l'année de CP, on observait: a) que les élèves initialement les plus faibles avaient effectivement un peu mieux progressé dans les classes ZEP que dans les classes témoin; b) que les différenciations (selon le sexe, l'origine sociale, la nationalitè, les acquis initiaux à l'entrée au CP) tendaient à être plus faibles dans classes ZEP et c) qu'en moyenne, les acquisitions scolaires étaient significativement inférieures dans les classes ZEP à celles des classes témoin.

Ce dernier résultat avait été expliqué par le fait que les élèves des classes ZEP avaient de fait eu un temps moindre consacré explicitement aux acquisitions scolaires alors qu'on analysait l'action engagée en termes d'investissement. Les moindres acquisitions en cours de CP seraient (plus que) compensées par des progressions ultérieures plus rapides comme conséquence de l'action " de fond" entreprise en CP.

2. Outre le fait que la scolarité au cours préparatoire ait été effectuée dans une classe ZEP ou dans une classe témoin "standard", il faut noter qu'on observe de fortes variations d'une classe à l'autre dans les acquisitions scolaires des élèves en fin d'année (4). Ces variations tiennent pour partie aux caractéristiques des élèves (origine sociale, géographique, ethnique, caractéristiques cognitives...) ainsi qu'à certaines caractéristiques agrégées du groupe classe (5) (niveau moyen, hétérogénéité, proportion d'élèves étrangers...). Cependant, après avoir tenu compte de ces facteurs de variations, il reste de très substantiels écarts d'acquisitions entre classes qu'il convient d'attribuer à des éléments, non étudiés dans cette recherche, qui caractérisent globalement la plus ou moins grande efficacité du maître dans la dimension des acquisitions scolaires des enfants. Ces écarts manifestent qu'au cours de leur première année de scolarité primaire des élèves de caractéristiques personnelles données sortent du Cours préparatoire avec des niveaux d'acquisition variés qu'on peut analyser comme des "plus " ou des " moins " par référence au niveau qu'auraient eu en moyenne des élèves de caractéristiques personnelles comparables.

Que ce soit donc en raison d'une activité pédagogique institutionnellement organisée telle que l'action ZEP dont nous avons rapidement décrit le fonctionnement ou de la relativement forte variabilité de l'efficacité pédagogique des maitres dans le cadre d'un fonctionnement institutionnel unifié, on observe que des élèves de caractéristiques personnelles données arrivent en fin de CP avec des niveaux d'acquisition assez fortement différents. Ces différenciations trouvent leur source du côté de l'offre 
scolaire, alors bien sûr que se combinent à ces différenciations celles qui sont propres aux caractéristiques personnelles des enfants.

Ici, nous nous intéresserons principalement aux différenciations qui trouvent leur origine dans l'offre scolaire lors de l'année de cours préparatoire en examinant leurs conséquences sur les acquisitions dans la scolarité ultérieure. Cette analyse apportera des éléments de faits dans le débat opposant les tenants de "l'acharnement pédagogique " et ceux pour qui une "pédagogie douce" et peu contraignante est finalement plus efficace.

\section{II. - LA StRUCtURE aNALYTIQUe RetenUe}

Elle consiste à représenter le processus temporel des acquisitions des élèves en spécifiant la structure causale des variables intervenant dans ce processus. Le schéma ci-après permet de visualiser les apports séquentiels et cumulatifs qui résultent dans les acquisitions en fin de CE2.

L'évaluation de l'école élémentaire doit en premier lieu, par nécessité, distinguer les différenciations interindividuelles qui se sont produites dans la période antérieure à l'entrée à l'école primaire et celles qui se sont générées pendant la scolarité élémentaire. Cette séparation entre ce qui s'est passé avant l'entrée au CP (qu'il est important pour nous de connaître mais qui constitue un domaine exogène à notre étude) et ce qui se constitue à l'école primaire est assuré par l'évaluation initiale des élèves effectuée au début du Cours préparatoire. D'une certaine façon, cette mesure évalue la "matière première » qui va être transformée par les activités pédagogiques de l'école primaire. On observe déjà de substantielles différences à l'entrée au Cours préparatoire dont une partie s'explique par des facteurs individuels liés à la personne de l'élève et une partie par les variables de son appartenance socio-démographique. II conviendra par conséquent de séparer dans les différenciations des acquisitions scolaires (en fin de CP et en fin de CE2) celles qui étaient préexistantes à l'entrée au CP de celles qui se sont constituées en cours d'enseignement primaire.

Ainsi la flèche marquée 3) sur le schéma permet-elle de mesurer l'impact des variables socio-démographiques à capacités scolaires initiales (Eln) données, c'est-à-dire l'impact de ces variables au cours de l'annee de CP sur les acquisitions des élèves en fin de cours préparatoire, alors que l'effet de ces mémes variables au cours de la période antérieure au CP est incorporé dans la variable caractérisant l'évaluation initiale.
a) $E \ln =f(C S D)$
[flèche 1)]
b) $E F C P=g(E I n, C S D$, Ped 1)
[flèche 2) + 3) + 4)]

L'effet total des caractéristiques socio-démographiques (CSD) sur les acquisitions scolaires en fin de CP (EFCP) résulte donc de deux effets temporels qu'il convient de bien différencier : un effet direct au cours du CP [flèche 3)] et un effet indirect qui transite par l'évaluation initiale (Eln) et qui a été constitué antérieurement au CP [combinaison des flèches 1) et 2)].

L'équation b) mesure aussi l'influence des variables pédagogiques au cours du CP (Ped 1). Par référence à une relation " moyenne " issue d'une pédagogie moyenne ou standard, on peut observer les déviations (en plus ou en moins) induites soit par une forme pédagogique particulière (par exemple classe ZEP/classe témoin) ou par chacun des maîtres titulaires d'un groupe classe.

De la même façon, en progressant temporellement dans la scolarité, on peut examiner les différenciations en matière d'acquisitions scolaires en fin de CE2 (EFCE). Celles-ci résultent d'un processus où les imbrications sont plus complexes. En effet, les acquisitions en fin de cours élémentaire résultent des acquisitions obtenues en fin de CP [elles-mêmes endogénéisées par l'équation b) ci-dessus] et des effets directs (en cours de scolarité CE1-CE2) des variables de l'évaluation initiale (Eln) [flèche 6)], des variables socio-démographiques (CSD) [flèche 5)] et des variables pédagogiques (Ped 1 et Ped 2) [flèches 8) et 9)]. On notera que la flèche 9) mesure les effets " contemporains" de la pédagogie du cours élémentaire sur les acquisitions à ce même niveau, alors que la flèche 8) mesure les effets différés lors des cours élémentaires de la pédagogie menée au cours préparatoire.

c) $E F C E=h(E F C P$, Eln, CSD, Ped 1, Ped 2) $[$ flèches 7) +5$)+6)+8)+9)]$

Le modèle structurel des équations simultanées $[a)+b)+c)$ ] peut être estimé directement avec des méthodes économétriques appropriées. Cependant, pour rendre les résultats plus facilement interprétables en référence à leur dimension pédagogique, il est apparu commode d'en proposer la transcription suivante:

Celle-ci porte essentiellement sur l'explication statistique de l'équation c), c'est-à-dire sur la variabilité des acquisitions en fin de CE2. En particulier, on modifiera la forme prise par l'influence des résultats de fin de Cours préparatoire, y compris celle des variables qui rendent compte de ces derniers.

Selon la transcription proposée, on distinguera 3 composantes dans les acquisitions scolaires en fin de CP :

1. Une première composante qui tient aux caractéristiques personnelles des élèves quant à leurs capacités générales d'acquisitions scolaires. Cette caractéristique préexiste à l'entrée au Cours préparatoire. 
Sexe

Age

Nationalité

CSP

Habitat

(1)

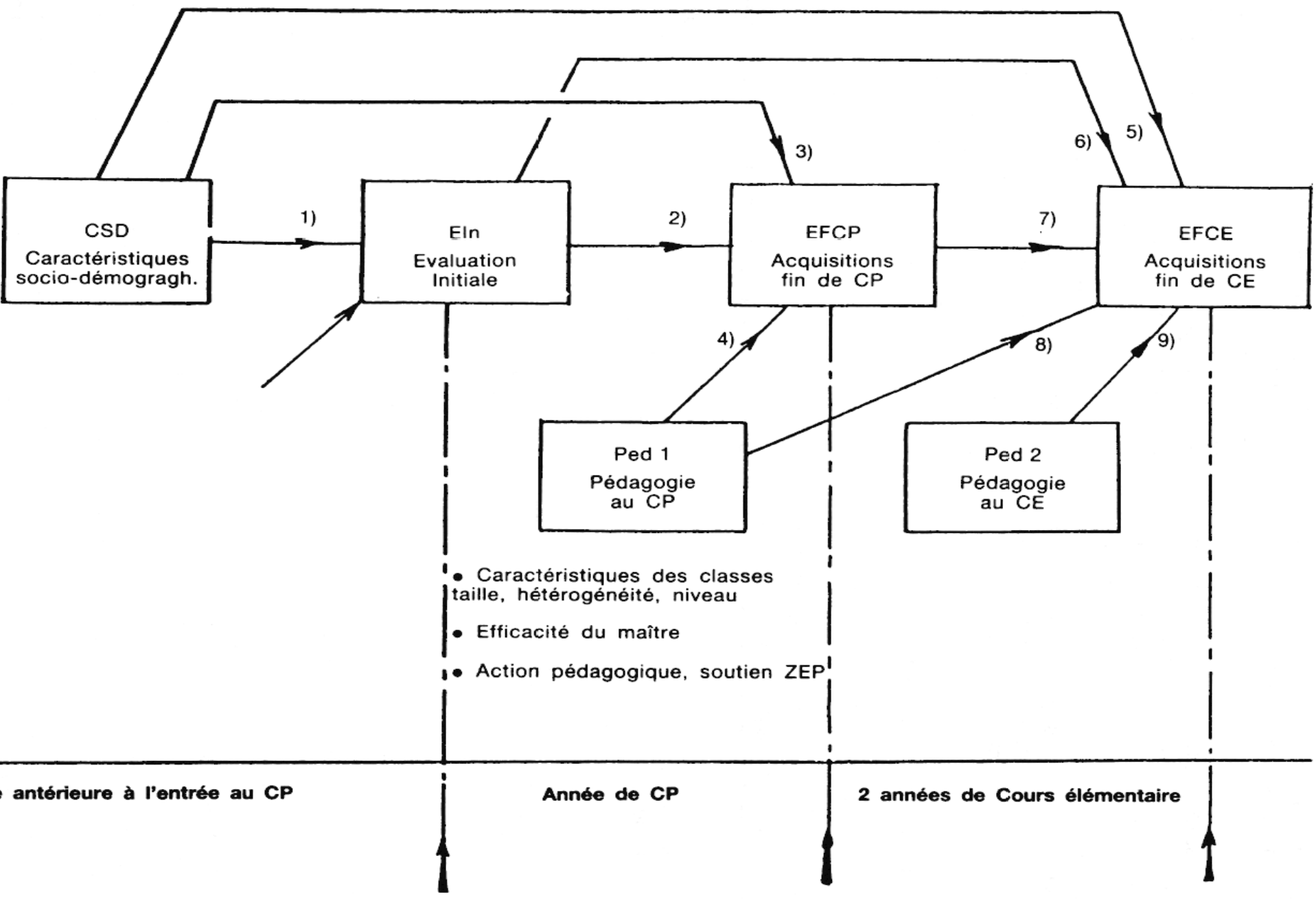

Evaluation initial à l'entrée au CP
Evaluation

fin de $C P$
Evaluation fin de CE2 
2. Une seconde composante qui est liée à l'efficacité des élèves à mobiliser leurs capacités générales pour les acquisitions effectives dans des domaines spécifiques. Cette seconde composante peut elle-même avoir une dimension générale d'adaptation à l'organisation concrète et aux situations d'enseignement et des dimensions disciplinaires spécifiques. Dans une large mesure, cette composante préexiste à l'entrée au Cours préparatoire mais c'est principalement au cours de cette classe qu'elle se révèle concrètement.

3. Une troisième composante qui est liée à la qualité de l'enseignement reçu par l'élève pendant son année de cours préparatoire. Plus l'enseignement de CP aura été de qualité, plus d'une part le niveau d'acquisitions scolaires en fin de cours préparatoire pour des élèves donnés sera élevé, plus d'autre part, seront positifs les effets différés sur les acquisitions lors de la scolarité ultérieure.

Les deux premières composantes sont liées à l'élève, alors que la troisième est liée à l'institution scolaire. La séparation des trois composantes dans les résultats de fin de CP n'est pas immédiate. Des estimations sont toutefois possibles.

On peut en effet partir de l'idée que le test initial (EIn) est une mesure de la première composante. On peut alors estimer conjointement les deux autres composantes en estimant la relation statistique entre le résultat de fin de $\mathrm{CP}$ et l'évaluation initiale.

$$
\text { 1) } E_{F C P_{i}}=\hat{b}_{0}+\hat{b}_{1} \cdot \operatorname{Eln}_{i}+\hat{U}_{i}
$$

Le résultat de fin de CP de l'élève i peut être écrit comme la somme de deux termes: d'une part le terme $\left(\hat{b}_{0}+\hat{b}_{1} \cdot\right.$ Eln) qui représente le résultat de fin de CP anticipé pour l'élève i compte-tenu de son score à l'épreuve du début de CP. D'autre part, le terme $\hat{U}_{i}$ (résidu estimé) qui représente tout ce qui dans le résultat de fin de CP ne peut être expliqué par le score initial. $\hat{U}_{i}$ représente donc de façon jointe les composantes 2 et 3 de la typologie proposée. On peut donc écrire:
- Caractéristiques personnelles générales préexistantes au $\mathrm{CP}$

- Effets de la pédagogie du CP

- Classes ZEP/non ZEP

- Effet maitre

- Caractéristiques personnelles d'adaptation à l'école révélées en cours de CP

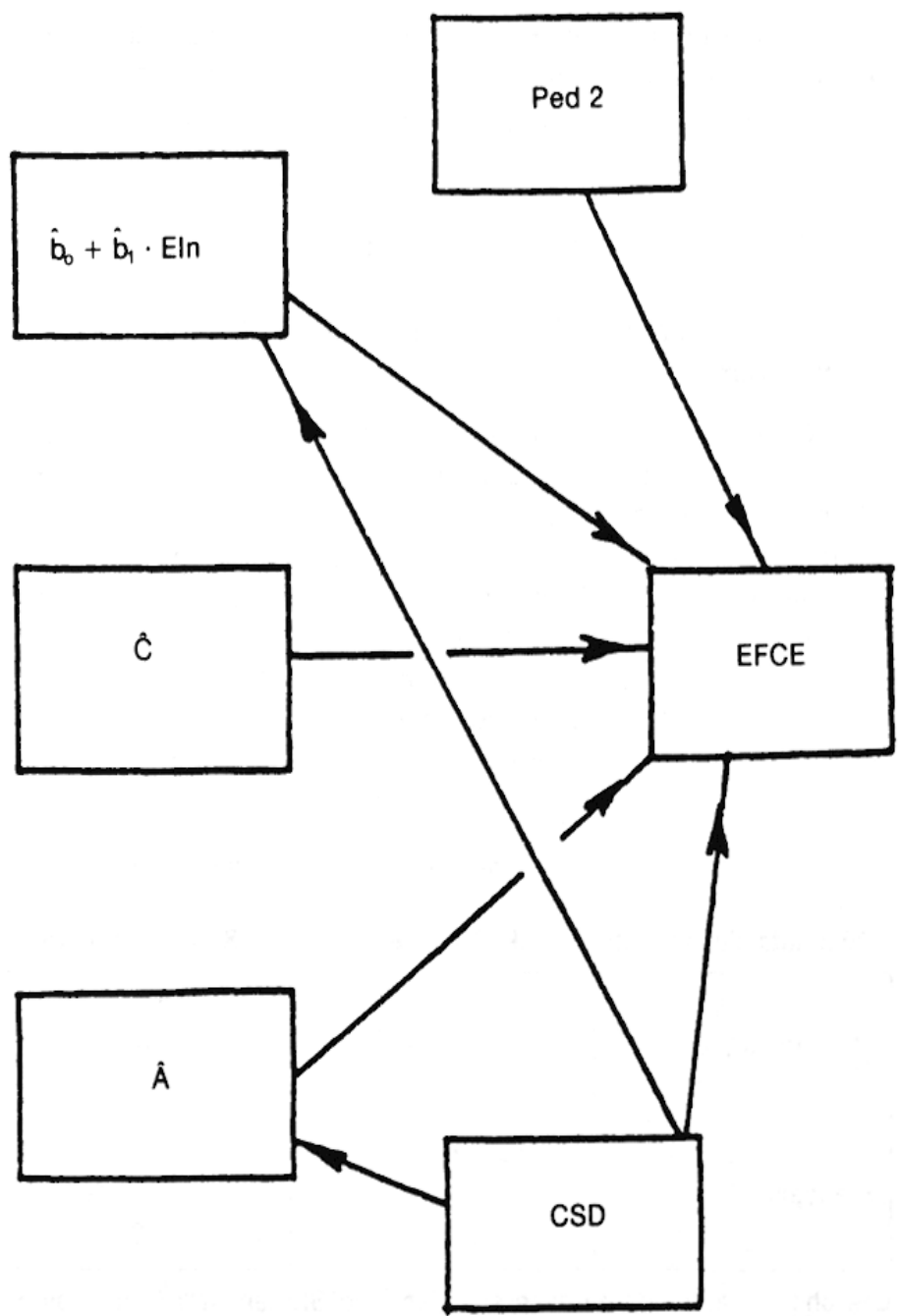


2) $\hat{U}_{i}=A_{i}+C_{i}$

dans laquelle $A_{1}$ représente les compétences générales d'adaptation à l'école (et les compétences particulières pour les différentes matières) de l'élève $i$, alors que $C_{i}$ représente la chance particulière qu'il a du d'être placé au cours de son année de $C P$ auprès d'un maître plus ou moins efficace pour les acquisitions scolaires.

Or $C_{i}$ peut être estimé directement par un jeu de variables muettes caractérisant l'appartenance à chaque groupe-classe dans un modèle expliquant le résultat de fin de CP après avoir contrôlé pour l'ensemble des variantes personnelles caractéristiques des élèves. Par différence entre ces valeurs estimées $\hat{C}_{i}$ et le terme $\hat{U}_{i}$ estimé précédemment, on déduit une valeur estimée $\left(\hat{A}_{i}\right)$ pour la composante $A_{i}$ recherchée.

Ayant distingué au sein des résultats de fin de CP ces trois composantes, on aboutit à un modèle beaucoup plus simple pour l'explication de la variabilité des résultats de fin de CE2. En outre, celui-ci se prête bien au test direct des types d'hypothèses présentées précédemment.

L'équation à tester (globalement ou en distinguant plusieurs dimensions - mathématiques, français... dans les acquisitions scolaires) est alors du type suivant :

3) $E F C E=f\left[\left(\hat{b}_{0}+\hat{b}_{1} \cdot E\right.\right.$ In $\left.), \hat{C}, \hat{A}, C S D, P e d 2\right]$

\section{III. - LES RÉSULTATS EMPIRIQUES OBTENUS}

\section{III.1. Les effets différés de l'action ZEP au cours préparatoire}

Nous avions observé dans l'analyse des résultats au cours préparatoire que globalement les acquisitions des élèves des classes ZEP étaient inférieures à celles des élèves des classes-témoin après avoir tenu compte des caractéristiques personnelles des élèves scolarisés dans les deux types de classes. Une hypothèse était alors que les élèves ayant bénéficié des activités dans le domaine des pré-requis (psychomotricité et langage) auraient effectué un "investissement " en ce sens qu'ils auraient consommé du temps au CP pour ces activités particulières (et corrélativement consacré moins de temps aux apprentissages classiques propres à la classe de cours préparatoire) mais que ceci les aurait mieux préparés pour les apprentissages futurs. Les bénéfices de cet investissement seraient donc différés et visibles seulement dans la période postérieure à la classe de CP.

Si cet argument était empiriquement valide, on devrait observer, à résultats de CP donnés, des progressions ultérieures significativement meilleures pour les élèves des classes ZEP et en particulier pour les élèves ayant bénéficié des activités de soutien.

Ce n'est pas ce qu'on observe. En effet, en contrôlant pour les caractéristiques socio-démographiques des élèves et leurs acquisitions en fin de $C P$, on observe que les élèves des classes ZEP ne progressent pas mieux en CE1-CE2 que des élèves comparables (en fin de $C P$ ) originaires des classes témoin. En fait, ils auraient même tendance à progresser moins puisque le coefficient de la variable muette ZEP dans le modèle estimé, bien que non significatif est néanmoins négatif.

Ce résultat, par conséquent, $n$ 'apporte pas de soutien empirique aux idées qui ont sous-tendu l'action ZEP dans les classes primaires des communes de Chenôve et Longvic. En effet, non seulement les acquis ont été en moyenne inférieurs au cours de l'année de cours préparatoire, mais là encore il n'y a eu, au mieux, aucun rattrapage dans les années ultérieures.

Ce résultat prend même davantage de force quand on examine les effets différés de l'action menée au CP en distinguant les différents groupes constitués dans les classes ZEP. En effet, les élèves ayant bénéficié d'un soutien (langage, psychomoteur ou mixte) ont tendance, et de façon significative, à progresser moins vite pendant les deux années de cours élémentaire. L'impact quantitatif net (pour les élèves comparables) n'est toutefois pas très important, il est cependant à la fois négatif et souvent significatif.

Le tableau ci-après donne les résultats obtenus:

Impact net (6) de l'action ZEP au CP dans les acquisitions au cours éfémentaire

\begin{tabular}{|l|c|c|c|c|c|}
\hline Résultats de fin CE2 & ZEP/Clas. témoin & Soutien langage & Soutien psychomoteur & Soutien mixte & Approfondissement \\
\hline \multirow{2}{*}{ Mathématiques (7) } & $-0,09(0,8)$ & - & - & - \\
\cline { 2 - 5 } & - & $-0,31(1,5)$ & $-0,28(1,4)$ & $-0,28(1,8)$ & $+0,06(0,2)$ \\
\hline \multirow{2}{*}{ Français (7) } & $-0,14(1,1)$ & - & - & - & - \\
\cline { 2 - 6 } & - & $-0,39(1,8)$ & $-0,26(1,7)$ & $-0,32(1,9)$ & $+0,03(0,3)$ \\
\hline
\end{tabular}

Les chiffres entre parenthèse sont les $t$ de Student (t 1,6 pour significativité au seuil de $10 \% ; t 1,96$ pour significativité au seuil de $5 \%$ ). 
II faut noter toutefois que les résultats obtenus concernent une action pédagogique particulière et ne peuvent donc pas avoir directement valeur de généralité. $\mathrm{Ce}$ ne sera pas le cas de l'analyse des effets différés des différenciations dérivant de la variabilité de l'efficacité pédagogique du maître dans une classe donnée (ici, le cours préparatoire), qui est à l'évidence un phénomène non spécifique à l'échantillon étudié. Abordons maintenant les résultats empiriques de cette analyse.

\section{III.2. Les effets différés aux cours elémentaires} de l'efficacité pédagogique des maîtres de CP

Nous avons déjà présenté quels étaient les facteurs associés aux acquisitions en cours de $\mathrm{CP}(8)$, nous nous attacherons ici à présenter les résultats concernant les acquisitions propres aux deux années de cours élémentaire. Nous proposerons uniquement les estimations du modèle structurel dans sa forme transformée à équation unique selon sa transcription finale présentée dans la section II de ce texte (équation 3) sachant que la variable cible de l'analyse est la variable $\hat{c}$, les autres, dont il est néanmoins intéressant d'analyser l'effet n'ont néanmoins que le statut de variable de contrôle (9).

1. Examinons en premier lieu l'influence des variables socio-démographiques pendant la scolarité des cours élémentaires $1^{\text {"0 }}$ et $2^{\circ}$ années. On retrouve ici le même type de structure que lors de l'analyse des facteurs ayant une influence nette sur les acquisitions scolaires en cours de $\mathrm{CP}$. En effet, on observe que les variables socio-démographiques exercent un impact à peu près nul sur les acquisitions dans le domaine des mathématiques alors que dans le domaine du français, les progressions sont significativement influencées par ces variables. Les filles progressent significativement mieux que les garçons (confortant ainsi leur avantage en fin de CP). Toujours en ce qui concerne les acquisitions en français en cours de $C E$, les enfants de cadres (et dans une moindre mesure d'employés) enregistrent des progressions significativement supérieures à celles des enfants d'ouvriers creusant ainsi les différenciations de fin de cours préparatoire.

Les acquisitions aux cours étémentaire (10)

\begin{tabular}{|c|c|c|c|c|c|}
\hline \multirow{2}{*}{\multicolumn{2}{|c|}{$\begin{array}{l}\text { Domaine d'acquisition en fin de CE2 } \\
\text { Variables }\end{array}$}} & \multicolumn{2}{|c|}{ Français } & \multicolumn{2}{|c|}{ Mathématiques } \\
\hline & & $\beta$ & $\mathrm{t}$ & $\beta$ & $t$ \\
\hline féminin & masculin & $-0,22$ & $3,9^{* * *}$ & 0,03 & 0,5 \\
\hline \multicolumn{2}{|c|}{ âge } & $-0,49$ & $4,8^{* * *}$ & $-0,32$ & $3,0^{\cdots}$ \\
\hline \multirow{3}{*}{ Français } & Etrangers $<5$ ans & $-0,06$ & 0,3 & 0,22 & 1,1 \\
\hline & Maghrébins $>5$ ans & 0,25 & $1,7^{*}$ & 0,08 & 0,5 \\
\hline & $\begin{array}{l}\text { Autres étrangers } \\
>5 \text { ans }\end{array}$ & 0,04 & 0,1 & 0,05 & 0,2 \\
\hline \multirow{3}{*}{ Père ouvrier } & Père cadre & 0,49 & $2,7^{* * *}$ & 0,18 & 1,0 \\
\hline & Père employé & 0,32 & $1,9^{* *}$ & 0,05 & 0,3 \\
\hline & Père "absent" & 0,12 & 0,5 & $-0,27$ & 1,1 \\
\hline \multicolumn{2}{|c|}{$\begin{array}{l}\text { Capacités personnelles générales } \\
\left(b_{0}+b_{1} \cdot \text { Eln }\right)\end{array}$} & 0,40 & $6,5^{\cdots}$ & 0,49 & $7,9^{\cdots}$ \\
\hline \multirow{2}{*}{$\begin{array}{l}\text { Capacités } \\
\text { d'adaptation ( } \hat{A})\end{array}$} & Français & 0,24 & $4,1^{\cdots *}$ & 0,19 & $3,3^{\cdots}$ \\
\hline & Mathématiques & 0,17 & $3,1^{\cdots *}$ & 0,29 & $5,1^{\cdots}$ \\
\hline \multirow{2}{*}{$\begin{array}{l}\text { Ecarts dus } \\
\text { aux maîtres (Ĉ) }\end{array}$} & Français & 0,18 & $2,3^{* *}$ & 0,03 & 0,4 \\
\hline & Mathématiques & 0,06 & 0,8 & 0,16 & $2,1^{*}$ \\
\hline \multicolumn{2}{|c|}{ Part de variance expliquée } & \multicolumn{2}{|c|}{0,49} & \multicolumn{2}{|c|}{0,46} \\
\hline
\end{tabular}

"Significatif au seuil de $10 \%$; "* seuil de $5 \%$; "** seuil de $1 \%$. 
Enfin, on retrouve un impact plus ou moins comparable à celui observé en CP pour les variables de nationalité. En général, les enfants d'origine étrangère ne progressent pas moins bien (tant en français qu'en mathématiques) que leurs homologues français de caractéristiques comparables par ailleurs. Les enfants d'origine maghrébine progresseraient même plutôt mieux.

2. Infiuence des différentes composantes des acquisitions au CP sur les acquisitions au CE. Les variables socio-démographiques dont nous venons d'examiner l'impact n'exercent toutefois qu'une influence globale limitée. En termes de part de variance expliquée, l'impact est inférieur à 0,12 dans la dimension du français et à 0,02 pour les mathématiques. C'est dire que leur influence s'exerce seulement à la marge d'un processus temporel d'acquisitions relativement fort pour l'ensemble de la population.

Dans ce processus temporel d'acquisitions, tel qu'il est décrit par le modèle, les caractéristiques personnelles des élèves jouent un rôle déterminant et une partie substantielle des différenciations dans les acquisitions en fin de cours élémentaire s'explique par des différences entre les élèves qui préexistent à l'entrée du cours préparatoire (11). Toutefois, au-delà de ce qui préexiste chez les élèves quand ils accèdent à l'école primaire ou qui se révèle chez eux en cours de $C P$, il reste qu'il subsiste des espaces de liberté non négligeables pour l'action pédagogique. Tout n'est pas inscrit dans les caractéristiques des élèves (exogènes pour l'institution puisque l'école est obligatoire et qu'il n'y a aucun mécanisme de sélection à l'entrée) et le fonctionnement de l'école joue aussi un rôle dans la production des différenciations inter-individuelles en matière d'acquisitions scolaires.

Nous avions observé (12), dans l'analyse de la variabilité des acquisitions scolaires au cours préparatoire que des élèves de caractéristiques personnelles initiales données étaient crédités d'acquisitions effectives très différentes en fin d'année selon le maître auprès duquel ils avaient été placés. Cette variabilité en soi n'aurait pas d'importance si elle ne constituait que des différenciations transitoires sans conséquence pour les acquisitions futures. L'analyse empirique effectuée sur les progressions ultérieures au CP pendant les deux années de cours elémentaire montre clairement qu'il n'en est pas ainsi et qu'il y a une substantielle rémanence de ces acquis.

Les résultats des estimations statistiques consignés dans le tableau ci-avant indiquent que les écarts nets dus au maître de CP restent bien visibles dans les acquisitions scolaires mesurées deux années après puisqu'ils y exercent un impact significatif (au seuil de $5 \%$ ) et quantitativement non négligeable (environ de 10 à $15 \%$ de la variance expliquée du résultat en fin de CE2).
Si on compare la valeur moyenne de ces écarts de fin de CE2 dus aux maîtres de CP avec leur valeur initiale de fin de cours préparatoire on observe qu'ils sont bien sûr réduits, mais restent néanmoins substantiels. Ils représentent $39,4 \%$ de l'écart initial pour les acquisitions en mathématiques et $37,2 \%$ pour le français.

Pour apprécier l'intensité de la rémanence de l'effet maître et d'une façon générale pour évaluer l'impact de la diversité de l'efficacité pédagogique des maîtres dans la genèse des différences interindividuelles en matière de réussite scolaire, il est sans doute utile de souligner que les influences respectives des caractéristiques du maître et de l'élève n'ont normalement pas le méme impact cumulatif au long de la scolarité de l'élève.

En effet, les caractéristiques personnelles de l'élève sont dans une large mesure permanentes dans le processus longitudinal d'acquisitions scolaires. Les élèves qui ont des caractéristiques personnelles favorables ont déjà des acquisitions plus élevées à l'entrée à l'école primaire ; ayant de meilleures bases et une meilleure fonctionnalité pour apprendre, les écarts par rapport aux élèves initialement plus faibles vont ainsi se creuser au fur et à mesure que le stock à acquérir augmente, d'année en année faisant ainsi accroître l'hètérogénéité interindividuelle des acquis.

Au contraire de l'élève dont les caractéristiques de base sont permanentes, un maître donné n'exerce son influence propre que pendant une durée limitée et s'il est vrai, comme nous l'avons montré pour le CP (13), que l'effet du maître peut être substantiel sur une année, celui-ci est par nécessité plus limité quand on considère les acquisitions sur plusieurs années. En effet, dans la majorité des cas (et en particulier dans tous les cas de l'échantillon étudié), l'élève a plusieurs maîtres successifs au cours de sa scolarité et s'il n'y a pas de corrélations fortes entre l'efficacité des maîtres en charge du même élève au cours d'années successives, alors tout se passe comme si l'élève avait un * portefeuille de maîtres n. Et au même titre que la constitution d'un portefeuille d'actions (pour ne pas mettre ses cufs dans un même panier) a une variance réduite par rapport à celle des actions individuelles qui le composent, l'effet global dû aux maîtres sur plusieurs années sera plus faible que celui correspondant à une année particulière.

Sur les trois premières années de l'école primaire, dans l'étude empirique dont on relate ici les résultats, et en supposant que l'effet-maître associé à une année scolaire soit identique en CP, CE1 et CE2 d'une part et qu'il n'y ait pas de corrélation entre l'efficacité pédagogique des maîtres successifs [hypothèse la plus plausible a priori (14)] d'autre part, alors on peut calculer que l'écart moyen dû aux maîtres dans les acquisitions en fin de CE2 
ne serait de $57 \%$ de l'écart moyen enregistré au cours d'une année donnée.

Ce long détour nous permet alors de mieux apprécier l'intensité de la rémanence de l'effet maître du CP dans les acquisitions en fin de CE2. En effet, nous savons maintenant que compte-tenu de l'effet-portefeuille lié à la succession des maîtres, on peut anticiper qu'une complète rémanence des écarts dus aux maîtres de CP conduirait à un écart à hauteur de $57 \%$ de cette valeur deux années après. Or la rémanence effective mesurée dans l'échantillon étudié est proche de $40 \%$; c'est dire qu'environ les deux-tiers des écarts initiaux dûs aux maîtres de CP sont encore visibles deux années après, ce qui constitue à l'évidence une valeur élevée.

\section{N. - CONCLUSION}

Au terme de la partie empirique de cette étude, il ne nous reste plus qu'à transcrire les résultats de l'analyse statistique en termes de "validation-réfutation " des corps théoriques présentés dans l'introduction de ce texte.

Le résultat le plus massif est que si au cours de l'année de CP, en raison d'une action pédagogique spécifique ou en raison de la variabilité de l'efficacité pédagogique du maître, on observe que des élèves acquièrent plus ou moins de connaissances scolaires (c'est-à-dire s'ils réalisent des "plus " ou des « moins " par rapport à ce qu'on aurait pu prédire compte-tenu de leurs caractéristiques personnelles), alors ces différences d'acquisitions ne sont pas transitoires mais auront des effets rémanents significatifs sur les acquisitions futures. Ceux des élèves qui auront eu la chance d'avoir, en CP, un maître plus efficace que la moyenne pour ce qui est de la transmission des connaissances scolaires, conserveront dans une large mesure ces acquis dans leurs deux années de scolarité ultérieure et progresseront mieux que ceux des élèves qui ont eu un maître moins efficace de ce point de vue en $C P$.

L'argument selon lequel ces écarts seraient transitoires et finalement de peu d'importance lorsqu'on étend la perspective temporelle à quelques années apparaît donc, sur les données analysées, clairement réfuté par les faits. II faut toutefois souligner que le test empirique a porté sur les effets différés des acquis de la classe de CP qu'on peut probablement considérer comme une classe particulièrement fondamentale et marquante pour la scolarité ultérieure. II serait donc souhaitable de ne pas donner aux résultats obtenus une généralité qu'ils n'ont pas pour d'autres niveaux scolaires.

II reste toutefois que ces résultats doivent apporter un soutien scientifique et des encouragements aux enseignants qui, par leur activité quotidienne, cherchent à rendre maximum les acquisitions de connaissances des élèves qui leur sont confiés. A ceux qui donnent moins d'importance aux acquisitions de connaissances scolaires, ces résultats soulignent, non pas que leur action n'est pas utile, car il est possible que les élèves progressent dans d'autres dimensions que celles des acquisitions de connaissances scolaires formelles, mais que si leurs élèves ont acquis moins, cela constituera pour ces derniers un handicap durable dans leur scolarité ultérieure.

Août 1986

Alain MINGAT

directeur de recherche CNRS-IREDU, Dijon
(1) Que les maîtres directeurs, d'ècoles, rééducateurs et psychologues scolaires en poste dans les écoles de l'échantillon étudié à Longvic et à Chenove, ainsi que les personnels de l'inspection départementale de Dijon 6 soient remerciés. Cette recherche n'existerait pas dans leur contribution. T. Vigneron a participé plus particulièrement à la production des résultats présentés ici.

(2) " La preuve du pudding, c'est quand on le mange *, pour donner une traduction libre d'un proverbe anglais.

(3) MINGAT A. - Evaluation analytique d'une action zone d'colucotion prioritaire au Cours properatoiro. Cahier de l'IREDU $n^{\circ}$ 39, septembre 1983 et * Les acquisitions scolaires des élèves au CP: Les origines des différences *, Revwe Francalos do Pidegoglo, n 69, octobre-décembre 1984.
(4) Ces différences sont observées à partir d'épreuves standardisées de connaissances et non à partir des résultats scolaires ou appréciations des maitres.

(5) Voir * Les acquisitions scolaires des blèves au CP ; les originales, les différences $"$, cit.

(6) II s'agit de l'impact net après contrôle des caractéristiques socio-démographiques, de l'évaluation initiale et aussi et surtout des resultats do fin de CP. Ces coefficients caractérisent donc bien les progressions au cours des deux dernières années de Cours élémentaire sur base de ce qui a été acquis en fin de Cours préparatoire. Comme les classes ZEP sont caractérisées par de moindres acquisitions nettes au CP, ces coefficients sont inferieurs a ceux qu'on obtient si on ne controle pas pour les acquisitions effectives on fin de CP, car 
on mesurerait alors l'effet cumulé des moindres acquisitions au cours de CP et de moindres progressions ultérieures. L'effet total est alors approximativement double de celui reporté dans le tableau ci-dessus.

(7) Les coefficients sont exprimés en unités d'écart-type de la distribution globale des scores de mathématiques et de français.

(8) RFP, octobre 1984, cit.

(9) C'est-à-dire de variables nécessaires pour que l'effet de la variable-cible soit estimé sans biais.

(10) Toutes les variables sont standardisées.

(11) Cette part est située quelque part dans la fourchette 30$60 \%$. La valeur $60 \%$ supposerait que la variance résiduelle du modèle s'expliquerait par des phénomènes aléatoires dont des erreurs de mesure sur les variables. Le chiffre de $30 \%$ supposerait que la variance inexpliquée par le modèle aurait pour origine l'oubli de variables importantes alors que spécification du modèle et mesure des variables seraient " parfaites . La réalité est entre ces cas extrêmes.

(12) RFP, 1984, cit.

(13) RFP, 1984, octobre-décembre, cit.

(14) Cette hypothèse est vraisemblablement vérifiée s'il y a changement de maitres chaque année scolaire. Ceci bien sûr n'est pas vrai si le maître suit ses élèves sur plusieurs années ou s'il y a "classe unique ". Dans ces cas, la corrélation de l'efficacité pédagogique des maîtres successifs est maximale et il n'y a pas réduction de l'effet-maître global sur plusieurs années. 